\title{
Equipos de reconstrucción provincial en Afganistán y el proceso de construcción de paz
}

\author{
Yuji Uesugi
}

\begin{abstract}
The toppling of the Taliban regime in Afghanistan has created great challenges to civilian organizations that seek to facilitate the process of post-conflict reconstruction and the establishment of a democratic state in this country. One of the main issues is the insecurity that still impacts many provinces and prevents the work of humanitarian or development organizations. In order to confront this problem, the Provincial Reconstruction Teams (PRTs) have been created. The teams are composed by civilian and military personnel and seek to advance projects beneficial to the Afghan population in regions, in which conventional civilian organizations still cannot operate. This paper presents a critical analysis of the work of PRTs, explains the reasons why they have been created, the criticisms that have been made by civilian organizations and the challenges they have to overcome if they are to be an effective tool in fostering Afghanistan's development.
\end{abstract}

\section{Introducción}

Los Equipos de Reconstrucción Provincial (PRT) ${ }^{1}$ realizan operaciones no-kinésicas ${ }^{2}$ por medio de un pequeño número de personal militar armado y personal civil de la comunidad diplomática y los organismos de desarrollo para la promoción del gobierno, la seguridad y el desarrollo en Afganistán tras los ataques del 11 de septiembre. El presente artículo arroja una luz sobre las funciones de los PRT como agentes de emergencia en el proceso de paz en Afganistán, y define a los PRT como organizaciones pequeñas que fueron (re) inventadas como una nueva forma de coordinación civil-militar a fin de maximizar efectos sinérgicos entre los distintos organismos que trabajan por la paz en este país.

1 Siglas en inglés de Provincial Reconstruction Teams (N. del ed.).

2 Del inglés "nonkinetic operations". Estas son misiones cuyo objetivo no es entrar en combate con fuerzas enemigas, sino ganar el apoyo de la población local (N. del ed.). 
Cuando el primer PRT fue establecido en Gardez, en noviembre de 2002, tal esfuerzo se consideró como una opción idiosincrática para la situación específica de seguridad en Afganistán, pero el 14 de noviembre de 2005 la Secretaria de Estado Condoleezza Rice hizo una aparición sorpresa en la inauguración del primer PRT en Irak anunciando que los Estados Unidos iban a establecer 15 PRT en ese país. ${ }^{3}$ Además, el 10 de enero de 2007, el Presidente George W. Bush anunció en su nueva estrategia que doblaría el número de PRT en Irak para ayudar a acelerar la transición de dicho país hacia la autosuficiencia. ${ }^{4}$

Si bien la noción de PRT ya ha sido ampliada más allá de la frontera de Afganistán, el presente documento se centra en las PRT afganas, las cuales ya han cumplido su quinto año desde la inauguración de la primera serie de PRT dirigidas por Estados Unidos, establecidas entre finales de 2002 y principios de 2003. Desde entonces, académicos, profesionales y responsables políticos en los Estados Unidos y Europa han presentado muchos argumentos a favor y en contra de la utilidad del modelo de PRT. En la primera aparición, los PRT recibieron un gran número de duras críticas de la comunidad de organizaciones humanitarias que operaban en Afganistán, pero una serie de recientes informes sobre su desempeño indicó su utilidad en diversos aspectos de la construcción de paz en Afganistán. ${ }^{5}$ Por ejemplo, uno de los más destacados comentaristas de los PRT sostiene que aunque la actual actitud de muchas ONG en el campo hacia ellos todavía se caracteriza por la desconfianza o la indiferencia, una posición de "pragmatismo de principios" ha surgido entre las ONG's. La comunidad de ONG's ha proporcionado retroalimentación a la contraparte militar con el objetivo de lograr una mayor sinergia entre el PRT y las organizaciones no gubernamentales. ${ }^{6}$ Sin embargo, estos argumentos se basan en evidencias anecdóticas y se presentan en ausencia de un entendimiento común de las actividades de los PRT y sus repercusiones en el proceso de paz en Afganistán. Cada vez menos acuerdan normas y medidas de eficacia, las cuales son necesarias para iniciar un

3 Polli Keller, "Condi Rice establece el primer PRT in Irak", Army News Service, noviembre 14, 2005.

4 "La Casa Blanca, Hoja informativa: El nuevo camino a seguir en Irak", <http://www.whitehouse.gov/ news/ releases/2007/01/20070110-3.html>, julio 14, 2008.

5 Para una revisión crítica, véase, por ejemplo: Save the Children, Equipos de Reconstrucción Provincial y humanitaria- las relaciones militares en el Afganistán (2004), y Stapleton, Barbara J., El equipo de reconstrucción provincial en el Plan de Afganistán: un nuevo rumbo?, Bonn, mayo de 2003. Para obtener una visión general, véase, por ejemplo, McNerney, Michael J., "Estabilización y Reconstrucción en Afganistán: son los PRT un modelo o un desorden?”, en: Parámetros, invierno de 2005-06, págs. 32-46; Jakobsen, Peter Viggo, PRT en: Afganistán: Exitosos, pero no suficiente, DIID Informe 2005, p. 6; y Dylan Hendrickson, Michael Bhatia, Mark Knight y Annabel Taylor, Una revisión del departamento de desarrollo internacional participación en equipos de reconstrucción provincial (PRT) en Afganistán , Departamento de Desarrollo Internacional (DFID), Londres, 8 de julio de 2005.

6 Barbara J. Stapleton, Presentación sobre Afganistán, Seminario Copenhague sobre Planificación y Acción Concertada de las Actividades Civiles y Militares en Operaciones Internacionales (20-21 de junio de 2005). 
diálogo fructífero entre perspectivas divergentes. ${ }^{7}$ Además, los debates actuales sobre los PRT no se basan en el actual discurso de coordinación civil-militar y la cooperación en operaciones de paz y estabilidad, a pesar de que los PRT son una forma de operación civil-militar adaptada a la situación en Afganistán.

Por lo tanto, este artículo tiene por objeto presentar un análisis de base para el desarrollo de un conjunto de criterios que permitan evaluar el desempeño de los PRT como mecanismo de coordinación civil y militar, haciendo referencia a un debate sobre la coordinación civil-militar y la cooperación en relación con los procesos de paz. ${ }^{8}$ Este documento busca ofrecer una revisión objetiva del concepto y del desempeño de los PRT como mecanismos de emergencia en el proceso de paz en Afganistán. De este modo, pretende contribuir a un debate actual sobre si los PRT son herramientas eficaces en operaciones de paz y estabilidad, y sobre si el modelo de PRT representa una nueva forma de coordinación civil-militar. En resumen, este artículo apoya el argumento de que los PRT necesitan un enfoque sistemático que permita la medición de su éxito.?

El interés de este ensayo es evaluar la eficacia de los PRT como herramienta en la consecución de los objetivos establecidos para ellos. En otras palabras, tiene por objeto examinar la eficacia de PRT como un instrumento para cerrar las brechas existentes en el proceso de paz en Afganistán. El Manual de PRT, preparado conjuntamente por la Fuerza de Asistencia para la Seguridad Internacional (ISAF), ${ }^{10}$ la Organización del Tratado del Atlántico Norte (OTAN), y la Misión de Asistencia de las Naciones Unidas en Afganistán (UNAMA), ${ }^{11}$ reconoce que el proceso de construcción de paz en Afganistán se enfrenta a un problema en la etapa media de transición, y muchas zonas del país parecen estar "estancadas" en ese punto. Es decir, mientras que las operaciones kinésicas ${ }^{12}$ han casi cesado en su mayoría, estas zonas no han avanzado significativamente y están en riesgo de recaer si se retiran las fuerzas de seguridad..$^{13}$ El Manual de PRT afirma que este problema existe debido a que a menudo no hay actores,

7 Perito, Robert M, “La experiencia de EE.UU. con los Equipos de Reconstrucción Provincial en Afganistán. Enseñanzas extraídas”, United States Institute of Peace, Informe Especial 152 (octubre de 2005).

8 Uesugi, Yuji, “Coordinación civil-militar y la cooperación en la asistencia humanitaria y operaciones de paz: sus posibilidades y desafíos” en: WAVOC (ed.), Paz (Tokio: WAVOC, 2005) pp. 11-19. Véase también, Uesugi, Yuji, "Naciones Unidas para el proceso de paz y el nexo entre establecimiento de conflictos y resolución de conflictos - Un estudio de caso comparativo de las Naciones Unidas en Chipre y Camboya”, Tesis de doctorado presentada a la Universidad de Kent en Canterbury, de agosto de 2003.

9 McNerney, Michael J., "Estabilización y reconstrucción en Afganistán: Son los PRT un modelo o un desorden?”, en: Parámetros, invierno de 2005-06, p. 39.

10 Siglas en inglés de International Security Assistance Force (N. del ed.).

11 Siglas en inglés de United Nations Assistance Mission in Afghanistan (N. del ed.).

12 Operaciones de combate (N. del ed.).

13 Manual PRT, 2a edición, octubre 31 de 2006, p. 5, párrafo 12. 
aparte de los militares, que puedan operar en zonas inestables. Para que los militares puedan relevar la responsabilidad a los actores civiles apropiados, deben primero entregar un cierto nivel de estabilidad en la zona para que los actores civiles puedan operar. Sin embargo, la misión de ayudar en esta transición está más allá de la experiencia y las capacidades de los militares. Tal experiencia reside en los organismos diplomáticos y de desarrollo, pero debido a la inestabilidad estos no pueden operar en estas áreas utilizando su programa tradicional de mecanismos de ejecución. ${ }^{14}$

Este problema es a menudo considerado como un dilema desarrolloseguridad. En la mayor parte de los esfuerzos de reconstrucción después de un conflicto, la brecha entre la seguridad y los enfoques de desarrollo socavan el sano progreso en la construcción de la paz. Como se mencionó claramente en el Informe del Desarrollo de los Objetivos del Milenio (MDG) ${ }^{15}$ de Afganistán en 2005, "el desarrollo sin seguridad es inalcanzable, y la seguridad sin desarrollo no tiene sentido". El proceso de construcción de paz en Afganistán parece haber caído en este dilema debido a la falta de coordinación y cooperación civilmilitar. ${ }^{16}$ De hecho, fue por esta razón que se inventó el concepto de PRT, los cuales fueron instalados como un mecanismo que podría resolver el problema dilema cerrando la brecha existente entre la seguridad y los enfoques de desarrollo. El Manual de PRT establece claramente que "un PRT es una institución civil-militar capaz de penetrar en las zonas más inestable e inseguras, gracias a su componente militar, y es capaz de estabilizar dichas zonas dada la combinación de capacidades de sus componentes diplomático, militar, y económico". ${ }^{17}$ En otras palabras, los PRT se introdujeron para resolver el dilema desarrolloseguridad mediante la creación de un marco que permitiera a los actores civiles y militares trabajar juntos en un entorno no permisivo, y así producir esfuerzos y efectos sinérgicos. Al mismo tiempo, los diseñadores del concepto de PRT intentaron resolver el problema de la coordinación civil-militar, o brecha civilmilitar, mediante la integración de componentes civiles y militares en una sola entidad. De hecho, los PRT son vistos por muchos como una estructura útil para coordinar los esfuerzos militares y civiles en la construcción de gobiernos estables e ideales. ${ }^{18}$

Este artículo se centra, por lo tanto, en los posibles puntos de referencia para evaluar la eficacia de los PRT, es decir, el desempeño de los PRT en el

14 Ibid. p. 5, párrafo 13.

15 Siglas en inglés de Millenium Development Goals (N. del ed.).

16 Reporte MDG de Afganistán, 2005.

17 Ibid. p. 5, párrafo 14.

18 Equipos de Reconstrucción Provincial en Afganistán: Una Evaluación Interinstitucional, 5 de abril de 2006, p. 23. 
proceso de cerrar las dos brechas principales existentes en la construcción de paz: la brecha civil-militar y la brecha desarrollo-seguridad. Este ensayo tiene por objeto evaluar el desempeño de los PRT abordando la siguiente pregunta de investigación: ¿han sido los PRT eficaces para cerrar la brecha civil-militar y la brecha seguridad-desarrollo en el proceso de construcción de paz en Afganistán? Antes de entrar a evaluar el desempeño de los PRT para cerrar estas brechas, se hará una breve descripción de la seguridad y el ambiente político de Afganistán después del 11 de septiembre, así como una descripción de la demografía de los PRT.

\section{Los PRT en Afganistán}

El 11 de septiembre de 2001 se recuerda como la fecha que cambió dramáticamente la imagen de la seguridad internacional. Estados Unidos atacó Afganistán, que estaba controlado por los talibanes, en retaliación por proporcionar refugio a Al-Qaeda, que se sospechaba estaba involucrada en el ataque del 11 de septiembre. El 7 de octubre de 2001 los Estados Unidos, junto con su aliado el Reino Unido, comenzó la "Operación Libertad Duradera” (OEF), y en el terreno la Alianza del Norte (una coalición de líderes militares y caudillos en contra de los talibanes) inició una serie de ataques contra los talibanes actuando en concordancia con la maniobra de la coalición. La Alianza del Norte derrocó a los talibanes en muchas partes del país con el apoyo de las fuerzas de la coalición y en noviembre de 2001 ocupó la capital, Kabul. Los talibanes fueron también derrotados en Kandahar y huyeron a la frontera con Pakistán. El colapso del régimen de los talibanes condujo al "Acuerdo sobre disposiciones provisionales en Afganistán en espera del restablecimiento de instituciones permanentes de gobierno", conocido como el Acuerdo de Bonn de 2001, por parte de los caudillos que estaban bajo la Alianza del Norte y otros grupos contra los talibanes que operaron fuera de Afganistán. En el Acuerdo de Bonn se define el proceso político para un nuevo Afganistán, y la promulgación de la constitución, seguida de las elecciones presidenciales y parlamentarias, puso en marcha el proceso político.

Las Naciones Unidas adoptaron lo que Lakhdar Brahimi llamó un enfoque de "huella ligera" y crearon una misión política civil: la UNAMA. ${ }^{19}$ Aunque la UNAMA no incluyó el componente militar en su organización, dos fuerzas

19 El mandato de la UNAMA incluye promover la reconciliación nacional, el cumplimiento de las tareas y responsabilidades encomendadas a las Naciones Unidas en el Acuerdo de Bonn, en particular las relacionadas con los derechos humanos, el Estado de derecho y las cuestiones de género, y la gestión de todas las misiones humanitarias, de socorro, de recuperación y reconstrucción actividades en Afganistán: <http://unama-afg.org/ about/overview.htm> 
militares internacionales operaban en Afganistán: una era la fuerza multinacional de la coalición dirigida por EE.UU bajo el mandato de la Operación Libertad Duradera (OEF), y la otra era la ISAF bajo mandato de las Naciones Unidas. La fuerza de la coalición seguía comprometida en la lucha contra los talibanes y contra Al-Qaeda en el sur y el Este del país. Inicialmente, la ISAF tenía cinco mil tropas en el terreno, provenientes de 19 países, y habían servido como fuerzas de paz, pero su responsabilidad se limitaba a proporcionar seguridad en Kabul. ${ }^{20}$

Si bien el régimen de Hamid Karzai parecía haber adquirido un cierto nivel de legitimidad a través del proceso político estipulado en el Acuerdo de Bonn, la situación de seguridad en Afganistán continúo como "no permisiva”, sobre todo en sus provincias remotas. Varios líderes militares mantuvieron el control de facto de la mayoría de las provincias de Afganistán, y los movimientos de insurgencia a cargo de los remanentes talibanes y de otras facciones no parecían acabarse. Han pasado más de 7 años desde la caída del régimen talibán en 2001, pero los EE.UU. y el gobierno de Karzai siguen haciendo arduos esfuerzos para contrarrestar la insurgencia. Numerosos informes de incidentes sobre problemas de seguridad y ataques de la insurgencia se pueden encontrar casi a diario en el Sur y el Este del país, aunque la situación ha sido relativamente tranquila en el Norte y el Oeste.

De acuerdo con los términos de referencia para la CFC y los PRT de la ISAF en Afganistán, aprobados por el Comité Directivo y Ejecutivo de los PRT el 27 de enero de 2005 y en los que se estipularon las funciones y misiones de los PRT que operaban bajo la fuerza de la Coalición y la ISAF, los PRT se formaron para ayudar al gobierno afgano a extender su autoridad, facilitar el desarrollo de un entorno estable y seguro en la zona de operaciones identificadas y permitir los esfuerzos de restablecimiento de seguridad en el sector (SSR). ${ }^{21}$ A los PRT no se les encomendó operar de manera activa en la creación de un entorno seguro, pero se esperaba que desempeñaran funciones de apoyo ayudando a las fuerzas de seguridad del gobierno afgano a cumplir con esa tarea.

Inicialmente los PRT operaron al mando de las fuerzas de la coalición, pero en octubre de 2003 el Consejo de Seguridad de la ONU autorizó la ampliación de la OTAN y la misión de la ISAF más allá de Kabul y los PRT comenzaron

20 La OTAN asumió el mando de la ISAF en agosto de 2003, y con la Resolución del Consejo de Seguridad de la ONU 1510 (13 de octubre de 2003) la ISAF comenzó a asumir un papel más amplio en el apoyo del gobierno afgano más allá de Kabul. En diciembre de 2005, la Fuerza Internacional contó con cerca de 9.200 tropas de 35 países (la OTAN en Afganistán- Ficha de Prensa <http://nato.int/issue/afghanistan/050816-factsheet.htm>. A partir del 2 de enero de 2007, las cifras de la ISAF aumentaron a 33.460 tropas de 37 países.

21 El mandato se puede encontrar en el Apéndice I de Barnett R. Rubin, Humayun Hamidzada y Abby Stoddard, El Afganistán de 2005 y el futuro: Perspectivas de mejora de la estabilidad del Documento de Referencia, Instituto Holandés de Relaciones Internacionales, abril de 2005.

(N. del ed: SSR son las siglas en inglés de Security Sector Reform). 
a operar bajo el mando de la ISAF.22 Cuando la ISAF concluyó su expansión a nivel nacional el 5 de octubre de 2006, todos los PRT fueron puestos bajo su comando. Al 10 de junio de 2008, 26 PRT están en funcionamiento, 12 de los cuales son operados por los EE.UU. y se encuentran desplegados principalmente en el Este. Los 14 restantes están dirigidos por Canadá, República Checa, Alemania, Hungría, Italia, Lituania, Países Bajos, Nueva Zelanda, Noruega, España, Suecia, Turquía, el Reino Unido, y se encuentran principalmente en el Sur, Norte y Oeste de Afganistán..$^{23}$

El concepto de PRT fue presentado por los Estados Unidos en noviembre de 2002, cuando los comandantes de la coalición comenzaron a preparar la transición de la Operación Libertad Duradera (OEF) de una fase de lucha a fases de estabilización y reconstrucción. ${ }^{24} \mathrm{El}$ concepto fue concebido para satisfacer exigencias contradictorias. Había una urgente necesidad de ampliar en las provincias la legitimidad de un recién instalado gobierno central y de mejorar la situación de seguridad fuera de Kabul, a fin de que la reconstrucción pudiera tener lugar en todo Afganistán y el pueblo afgano pudiera apreciar los dividendos de la paz. Se reconoció que un entorno seguro podría ofrecer oportunidades para un mayor desarrollo y éste, a su vez, podría mejorar la seguridad del entorno. Al mismo tiempo, sin embargo, los Estados Unidos no podían darse el lujo de desplegar una fuerza robusta de mantenimiento de la paz en todo el país, ya que sus fuerzas estaban atareadas en la guerra de Irak. Además, los funcionarios de EE.UU. aseguraron que una gran presencia de tropas extranjeras sería contraproducente en la consecución de seguridad pública y apoyo para Afganistán. ${ }^{25}$

En tales circunstancias, el concepto de PRT se ha desarrollado esencialmente como un instrumento de ayuda a la transición. Se tenía la intención de que fuera una estrategia "no interventora" a través de la cual se pudiera mejorar la capacidad del nuevo gobierno afgano para regirse a sí mismo, mejorar la seguridad del sector afgano para proporcionar y mantener la estabilidad y la seguridad en el país, y mejorar las instituciones afganas que conllevaran a un desarrollo sostenible a largo plazo. Todo esto con el fin de que la participación

22 En cuanto a la Coalición de PRT, no hay fundamento jurídico específico para la autorización de tales esfuerzos. La Resolución del Consejo de Seguridad de Naciones Unidas 1510 (2003) autorizó, en virtud del Capítulo VII de la Carta de las Naciones Unidas, la ampliación del mandato de la ISAF en apoyo de la Autoridad de Transición Afgana en el mantenimiento de la seguridad en las zonas de Afganistán fuera de Kabul. En otras palabras, los PRT dirigidos por la OTAN o por la ISAF, que forman parte de la ISAF, son, en efecto, operaciones autorizadas por la ONU en el Capítulo VII.

23 ISAF hechos y cifras clave, Placemat: <http://www.nato.int/ISAF/docu/epub/pdf/isaf_placemat. pdf>, (14 de julio de 2008).

24 Jakobsen, Peter Viggo, PRT en Afganistán: exitosos, pero no suficiente, DIID Informe 2005, p. 11.

25 McNerney, Michael J., "Estabilización y reconstrucción en Afganistán: son los PRT un modelo o un desorden?”, en: Parámetros, invierno de 2005-06, p. 32 y p. 43. 
de los Estados Unidos dejara de ser necesaria. En resumen, los PRT intentaron abordar simultáneamente las tres dimensiones clave de la consolidación de la paz: el gobierno, la seguridad y el desarrollo. Los PRT fueron diseñados para generar efectos sinérgicos en tres organismos importantes del área: la diplomacia, la defensa y el desarrollo (a menudo conocidos como las “3D”). Los PRT adoptan un enfoque de inter-organismos 3D para abordar un reto tridimensional en la construcción de la paz. ${ }^{26}$ De hecho, el concepto de PRT proveyó una manera de facilitar la integración de los tres organismos esenciales para la construcción de paz y presentó un modelo para institucionalizar este tipo de enfoque de inter-organismos 3D.

\section{Brecha civil-militar en Afganistán}

El gobierno de los EE.UU. publicó una evaluación de los PRT titulada Grupos de Reconstrucción Provincial en Afganistán: Una Evaluación Interinstitucional (en adelante, la Evaluación), la cual identificó tres criterios para medir la eficacia de los PRT, incluyendo la coordinación civil-militar. ${ }^{27}$ Según la evaluación, los PRT comandados por los Estados Unidos tuvieron las siguientes deficiencias en lo relacionado a su desempeño en la coordinación civil-militar:

- La falta de directrices explícitas conllevaron a la confusión de las funciones civiles y militares en los PRT dirigidos por Estados Unidos.

- El comandante militar de los PRT dirigidos por Estados Unidos necesitaba incorporar proactivamente un representante externo al Departamento de Defensa (DOD) que operara en las decisiones sobre el liderazgo o metas de los PRT.

- La escasez de personal, la limitada capacidad técnica y de gestión de apoyo en Kabul, y la inadecuación de los mecanismos para la ejecución del proyecto socavaron la eficacia de los PRT dirigidos por Estados Unidos.

- A medida que el centro operativo para la reconstrucción y el gobierno se trasladó a las provincias, los programas de apoyo del Gobierno de los EE.UU. no pudieron mantener el ritmo.

- Se probó que era esencial el entrenamiento de grupos combinados de personal militar y civil.

26 Por cierto, este enfoque de PRT 3D accidentalmente coincide con la estrategia del gobierno japonés de asistencia para la reconstrucción de Afganistán - enfoque de los tres pilares para la "consolidación de la paz": proceso de paz (diplomacia), seguridad nacional (defensa), y reconstrucción y asistencia humanitaria (desarrollo).

27 Evaluación, p. 12. 
Esta evaluación ignora el actual debate sobre las relaciones civiles-militares y no logra resolver los problemas de dilema humanitario. La comunidad humanitaria en general tiene gran preocupación por borrar la distinción fundamental entre militares y no militares. No obstante, los organismos humanitarios comenzaron a enfrentarse con crecientes retos operativos, así como con mayores riesgos y amenazas para sus trabajadores en el campo, y tal realidad en el terreno ha exigido gradualmente diversas formas de coordinación civil-militar para las operaciones humanitarias. ${ }^{28} \mathrm{Si}$ bien esta evaluación se centró en la relación interinstitucional dentro del gobierno de los EE.UU., el verdadero desafío existía dentro de los PRT en sí, y entre los PRT y una comunidad amplia de ayuda. Una de las principales fuentes de tensión entre los PRT y la comunidad humanitaria ha sido la concepción por parte de la comunidad humanitaria de que el concepto de PRT viola los principios de asistencia humanitaria.

\section{Tensiones entre civiles y militares}

En las primeras etapas de la evolución de los PRT, el Órgano de Coordinación Institucional para el Auxilio a Afganistán (ACBAR), ${ }^{29}$ una organización de cobertura para organizaciones no gubernamentales que operan en Afganistán, hizo una serie de comentarios y recomendaciones pertinentes, constructivos y realistas con respecto al desempeño de los PRT. Por ejemplo, en una ocasión ACBAR sostuvo que:

Recomendamos el rápido desarrollo y la rápida aplicación de planes que anclarán todos los PRT exclusivamente en el área de Reforma del Sector de Seguridad. Concretamente los PRT deberían centrarse en apoyar el proceso de DDR y el entrenamiento del Ejército Nacional Afgano y la policía. Recomendamos poner fin a la participación de PRT en los proyectos de tipo asistencia humanitaria, y que se trasladen a la selección de proyectos que se centran en medidas prácticas para fortalecer la autoridad del gobierno provincial, como la reconstrucción de las estaciones de policía, aduanas y las oficinas administrativas locales. Con este fin, hacemos un llamado para cambiar las funciones de los PRT, que debe ser definido con claridad y precisión. El nombre de los PRT debería cambiarse por Grupos de Estabilidad Provinciales, para una mayor claridad. ${ }^{30}$

28 "Relaciones civiles y militares en situaciones de emergencia complejas. Un documento de referencia", Comité Permanente entre Organismos, 28 de junio de 2004, párrafo 1.

29 Siglas en inglés de Agency Coordinating Body for Afghan Relief (N. del ed.).

30 ACBAR, "Equipos de Reconstrucción Provincial y la situación de seguridad en Afganistán”, ACBAR Resumen de Política, (24 de julio de 2003). 
Esta lista de recomendaciones indica que las preocupaciones de la comunidad humanitaria con respecto a los PRT giran en torno al hecho de que el los PRT son un conjunto civil-militar que implica actores militares, políticos y agentes de desarrollo, y que algunos PRT han asumido la entrega de ayudas humanitarias y proyectos de rápido impacto (QIPs) ${ }^{31}$ a la población local. Aunque la mayoría de las recomendaciones fueron implementadas por los PRT, se mantuvieron diversas tensiones entre los PRT y la comunidad humanitaria en Afganistán, tales como la orientación de los PRT hacia operaciones de "corazones y mentes", y la preocupación de la comunidad humanitaria por la pérdida del espacio humanitario. De hecho, la participación de los PRT en la entrega de ayudas humanitarias y proyectos rápidos de impacto ha sido el principal punto de discordia, ya que borra los límites entre las organizaciones humanitarias y las militares.

\section{Principios rectores de los PRT}

Estas tensiones entre civiles y militares existentes en Afganistán fueron causadas por las evidentes diferencias entre algunos principios de funcionamiento de los organismos humanitarios y de las organizaciones militares. Las directrices existentes defendían la separación de los ámbitos humanitarios y los ámbitos de fuerzas armadas, y admitían operaciones conjuntas entre civiles y militares sólo como un último recurso. Por ejemplo, la sección "Operaciones de auxilio conjuntas entre civiles y militares" del documento "Relaciones entre civiles y militares en emergencias complejas" (documento de referencia del Comité Permanente entre Organismos IASC), de estableció lo siguiente. ${ }^{32}$

- Cualquier operación emprendida conjuntamente por los organismos humanitarios y fuerzas militares pueden tener un impacto negativo en la percepción de "imparcialidad y neutralidad" de los organismos humanitarios, y por tanto, afectar su capacidad para funcionar eficazmente a lo largo de una situación de emergencia compleja. Así, cualquier cooperación conjunta entre civiles y militares debe ser determinada por una evaluación exhaustiva de las necesidades reales del terreno y una evaluación de la capacidad de asistencia humanitaria civil para responder a ellas de manera oportuna. En la medida en que las operaciones conjuntas con los militares no pueden ser evitadas, podrán emplearse sólo como un último recurso, y deben adherirse a los principios previstos en las Directrices sobre el Uso de Recursos 
Militares y de Defensa Civil para Apoyar Actividades Humanitarias de las Naciones Unidas en situaciones de Emergencia Complejas (Directrices MCDA).

- Uno debe ser consciente de que los militares tienen objetivos, intereses, horarios y prioridades diferentes a los de la comunidad humanitaria. Las operaciones de socorro prestadas por las fuerzas militares podrían estar condicionadas y pueden cesar cuando la misión de las fuerzas militares cambie, cuando estas últimas se muevan o cuando la población asistida no coopere con ellas. Esta acción por parte de los militares también puede llevarse a cabo basándose en las necesidades y objetivos de la fuerza y su misión, antes que en las necesidades de la población local.

Por otro lado, los Principios Rectores de los PRT, que abogan por la integración militar-civil para generar la unidad de esfuerzos entre los diversos agentes del terreno, violan las actuales directrices civiles-militares. Los Principios Rectores de los PRT tienen seis componentes: (1) se centran en el mejoramiento de la estabilidad, (2) funcionan como una organización integrada por civiles y militares (3) trabajan para un propósito común o estado final uniendo esfuerzos, (4) garantizan que los proyectos no dupliquen el trabajo de los demás, (5) sientan las bases para los cambios sostenibles a largo plazo, y (6) son conscientes de respetar las sensibilidades entre militares y civiles. El segundo punto de los Principios Rectores de los PRT no parece coincidir con la doctrina de la CIMIC OTAN (Centro de Excelencia para la Cooperación Civil-Militar), a pesar de que todos los PRT ahora operan bajo la el mando de la OTAN y la ISAF. La OTAN reconoce que sus esfuerzos deben evitar repercusiones negativas en la labor de las organizaciones humanitarias, así como en su neutralidad e imparcialidad. Si bien la mayoría de los elementos de los Principios Rectores de los PRT permanecen dentro de los parámetros de las actuales directrices para las relaciones entre civiles y militares, el enfoque fundamental de la "integración" entre civiles y militares en concepto de los PRT contradice el argumento central de "separación" en las directrices existentes.

\section{Cerrando la brecha civil-militar}

Esta brecha en las relaciones fundamentales civiles-militares, entre la "separación" de la que habla la comunidad humanitaria y la "integración" de la que hablan los PRT puede ser representada por el debate sobre el "espacio humanitario" o la eficacia de adherirse a los principios humanitarios, y el estab- 
lecimiento y mantenimiento del acceso a las personas necesitadas de asistencia humanitaria.

En general, es importante preservar el "espacio humanitario", que ha sido fundamental para el eficaz funcionamiento de los organismos humanitarios en un medio ambiente no permisivo. Por lo tanto, la preocupación de la comunidad humanitaria por la pérdida de tal "espacio humanitario" se debe abordar de manera adecuada si los PRT resultan eficaces a la hora de cerrar la brecha civil-militar. Al mismo tiempo, sin embargo, se debe reconocer el hecho de que los PRT fueron creados para satisfacer necesidades específicas y retos que se manifestian en el proceso de construcción de paz en Afganistán, uno de los cuales puede ser la brecha seguridad-desarrollo. En otras palabras, es importante encontrar una óptima combinación de interacciones civiles-militares en la que tanto la cuestión del "espacio humanitario" y la cuestión de la brecha seguridad -desarrollo puedan abordarse adecuadamente.

Una de las claves para encontrar tal combinación y hallar una respuesta al debate civil-militar sobre el "espacio humanitario" se encuentra en un enfoque centrado en el ámbito local, presentado por Jane Barry: "el debate civil-militar debe ser reajustado y centrarse en primer lugar en las personas necesitadas de una respuesta humanitaria". ${ }^{33}$ La comunidad humanitaria se ha adherido a los principios humanitarios, ya que considera que este enfoque le permite mantener su capacidad de funcionar de manera efectiva a lo largo de una emergencia compleja. El enfoque centrado en el ámbito local nos recuerda que la regla más importante para la comunidad humanitaria es que puede mantener el acceso a las personas vulnerables de manera que pueda salvarlas y ayudarlas. Es evidente, sin embargo, que la adhesión estricta a los principios humanitarios no siempre garantiza un espacio humanitario para los organismos humanitarios en Afganistán. Con el fin de establecer y mantener el espacio humanitario de manera efectiva en el terreno, es importante comprender lo que constituiría los cimientos de tal espacio en cada caso, ya que pueden variar en cada circunstancia. ¿Es la imparcialidad política de los organismos humanitarios un factor esencial en la creación de “espacio humanitario"? ¿Se está borrando la línea límite entre los organismos humanitarios y una cierta organización militar que opera en la escena, en detrimento de la preservación de todos los "espacios humanitarios" de la

33 Barry, Jane y Anna Jefferys, "Un puente demasiado lejos: los organismos de ayuda y los militares en la respuesta humanitaria”, Documento NPD 37, p. 15. 
zona? ${ }^{34}$ Si la respuesta a estas preguntas es afirmativa, entonces los PRT tendrán que mantener una clara distinción entre ellos y los organismos humanitarios.

Por otra parte, si la adhesión estricta a los principios humanitarios de neutralidad e imparcialidad no garantiza el acceso seguro a las personas vulnerables que necesitan asistencia, entonces se podría considerar la idea de una cooperación civil y militar y/o incluso operaciones conjuntas entre civiles y militares. Si la eficacia y la eficiencia de la ayuda son fundamentales para la creación de un espacio humanitario, y si la cooperación con los militares mejora la capacidad de los organismos humanitarios para proveer ayuda, entonces la cooperación civil y militar es una forma de progresar y los PRT pueden cerrar la brecha civil-militar. Si los ataques indiscriminados como los atentados suicidas y de IED (dispositivo explosivo improvisado) por parte de grupos terroristas y los ataques deliberados contra los trabajadores de ayuda humanitaria por parte de delincuentes causan la pérdida del espacio humanitario, entonces se puede considerar la alternativa de escoltas armados que pueden operar en un espacio humanitario "voluntario" para los organismos humanitarios, pero no es seguro que los PRT puedan servir como un "vehículo" eficaz para reabrir enérgicamente el espacio humanitario.

La Evaluación señala que los PRT pueden ser una herramienta eficaz para cerrar la brecha civil(humanitaria)-militar en situaciones en las que los organismos humanitarios no pueden funcionar debido a la pérdida del espacio humanitario. ${ }^{35} \mathrm{Si}$ bien algunos PRT, en cierta medida, han demostrado tener éxito en este esfuerzo, la falta de capacidad suficiente y recursos civiles en los PRT, junto con la mala relación entre estos últimos y la comunidad humanitaria han impedido ejercer su pleno potencial como mecanismo para el cierre de brechas. Este punto también fue reconocido por la Evaluación: los PRT carecieron de los recursos necesarios (conocimientos técnicos y financiación civil), sufrieron una escasez de personal, limitada capacidad técnica y apoyo de Kabul (es decir,

34 La cuestión difícil aquí consiste en qué forma se debería responder a la situación en la que las operaciones de socorro apoyadas por los militares podrían salvar más vidas y aliviar el sufrimiento de manera más eficaz que las operaciones humanitarias realizadas únicamente por los organismos proveedores de ayuda humanitaria. $\chi^{A}$ quién se debería autorizar para hacer tan duro llamado de atención, y quienes deberían ser los responsables de las muertes y sufrimientos que podrían haberse evitado si a los militares se les permitiera violar los principios humanitarios y se les permitiera trabajar conjuntamente en las actividades de los organismos humanitarios? Esto conlleva a un juicio de valor: ¿A qué debería dársele prioridad: a la eficacia en salvar vidas en la actual crisis aunque haya que violar los principios, o a la eficacia en salvar vidas en otras situaciones de crisis mediante el cumplimiento de los principios? En otras palabras, hay que encontrar un buen equilibrio entre las necesidades a corto plazo de salvar vidas en una situación de emergencia y los requisitos a largo plazo de preservar la eficacia del régimen humanitario.

35 Evaluación, p. 6. 
los PRT carecían de donaciones de recursos), y han contado con un mecanismo insuficiente para la ejecución de proyectos. ${ }^{36}$

Los PRT también pueden ser una valiosa herramienta para cerrar la brecha civil(humanitario)-militar, incluso en la situación en la que los organismos humanitarios estén funcionando de manera eficaz, realizando funciones complementarias tales como el desarme, desmovilización y reinserción (DDR) y el restablecimiento de la seguridad (SSR), y estén apoyando grandes proyectos de infraestructura que sirvan, a largo plazo, como catalizadores para el desarrollo sostenible. De hecho, este efecto complementario de los PRT también puede influir en el cierre de la brecha seguridad-desarrollo que se revisará en la próxima sección. Sin embargo, los PRT carecían de comunicación y coordinación suficiente con los actores civiles externos que operaban fuera de su área pero que estaban en la misma zona, como la UNAMA, los organismos donantes bilaterales y ONGs que, de hecho, socavaban el efecto complementario de los PRT. Este punto fue destacado en la Evaluación, indicando que muchos programas de ámbito nacional que existían en las provincias tenían una mala coordinación con los PRT. ${ }^{37}$

\section{Brecha seguridad-desarrollo en Afganistán}

\section{Amenazas a la seguridad}

Las amenazas a la seguridad y estabilidad de Afganistán se pueden dividir en dos. En primer lugar, Afganistán se enfrenta a amenazas por la rivalidad entre tribus y líderes militares, y por la existencia de grupos criminales, incluyendo a los traficantes de droga. Contra esas amenazas, la construcción y fortalecimiento de la capacidad del aparato del Estado afgano para hacer frente a problemas internos de seguridad pública es un camino a seguir. Se ha iniciado el desarrollo de la capacidad de las fuerzas de seguridad afganas - Ejército Nacional Afgano (ANA) y la Policía Nacional Afgana (ANP) - en el marco del restablecimiento de la seguridad (SSR) del G-8. Estados Unidos ha tomado el liderazgo en el entrenamiento del ANA, y Alemania se ha comprometido con la reforma de la ANP. Al mismo tiempo, es también importante debilitar la fuerza de los caudillos, o líderes militares, y los grupos delictivos a través del desarme, desmovilización y reinserción (DDR) de los combatientes, y la disolución de grupos 
armados ilegales (DIAG). ${ }^{38}$

La otra fuente de amenazas proviene de las fuerzas de insurgencia contra el gobierno y de las organizaciones terroristas. Contra este tipo de amenazas, el restablecimiento de la seguridad (SSR) ha demostrado ser una medida útil, ya que el medio más eficaz contra la insurgencia es la creación de un entorno en el que la población local se sienta segura y protegida por el Estado. De hecho, la gente necesita tener confianza en el actual régimen y en que los talibanes u otro tipo de insurgencia no derrocará al gobierno. Esto si estamos de acuerdo en que mantener el actual marco político es la mejor opción disponible para el proceso de construcción de paz en Afganistán. Por lo tanto, es vital que la comunidad internacional mantenga su firme compromiso de apoyar al gobierno.

Otra manera eficaz de contrarrestar las amenazas de fuerzas que luchan contra el gobierno sería aumentar la legitimidad del gobierno afgano, así como su eficacia para prestar servicios a la población. El aumento del apoyo local a la administración central del gobierno puede crear fortalezas que operarán en contra de la insurgencia. Sin embargo, el proceso de construcción de paz en Afganistán se enfrenta a un gran reto: con el fin de proporcionar ayuda para el desarrollo, se debe proveer seguridad siempre, pero para mejorar la seguridad, la ayuda para el desarrollo debe mejorar las condiciones de vida del pueblo.

\section{La brecha seguridad-desarrollo}

La brecha seguridad-desarrollo surgió como resultado de la ausencia de seguridad y desarrollo en Afganistán luego de la caída del régimen talibán. En primer lugar, los ataques dirigidos por los Estados Unidos contra los talibanes crearon una ausencia de poder en el país. El gobierno afgano no tenía capacidad suficiente para llenar ese vacío. En lugar de ello, los caudillos, o líderes militares, pronto llenaron tal ausencia y comenzó el control de las provincias. La comunidad internacional creó la ISAF para ayudar a las fuerzas del gobierno afgano en la preservación de la seguridad, pero inicialmente la zona de responsabilidad se limitaba a Kabul y sus alrededores (UNSCR 1386, 20/12/01). Por supuesto, se llevaron a cabo acciones de DDR y SSR (reforma del ANF y de la ANP) para hacer frente a este desafío, pero la influencia del gobierno central siguió siendo limitada en las cercanías de Kabul y la situación de seguridad en las provincias (especialmente en el Sur y el Este) se mantuvo volátil.

Esta ausencia de poder se vio agravada aún más por los ataques indiscriminados, como de IED (dispositivo explosivo improvisado), o los ataques deliberados contra los trabajadores de ayuda humanitaria por parte de Al-Qaeda, las 
fuerzas insurgentes y entes criminales. Esto se tradujo en la pérdida del "espacio humanitario" en muchas partes de Afganistán. La falta de seguridad y la pérdida de espacio humanitario impidieron la labor de los organismos de ayuda civil. El aumento de las amenazas de las fuerzas talibanes impidieron a los organismos de ayuda civil trabajar en el Sur y Sureste de las provincias donde la asistencia humanitaria y el desarrollo eran más necesarios, no sólo desde la perspectiva de las necesidades humanitarias, sino también desde la perspectiva de la resolución del dilema seguridad-desarrollo. El pueblo debe percibir los "dividendos de la paz”, de lo contrario el actual gobierno perderá su apoyo, lo que puede crear condiciones perfectas para la operación de fuerzas de la insurgencia, como los talibanes, socavando así el esfuerzo del gobierno por mejorar la seguridad.

\section{Cerrando la brecha seguridad-desarrollo}

Aunque los planificadores de la seguridad en el Pentágono parecían haberse dado cuenta de que la victoria militar en el campo de batalla debe ser continuada con vigorosos esfuerzos de desarrollo, y así cerrar la brecha seguridad-desarrollo y ganar la paz en Afganistán, sabían también que la victoria militar no es el fin. El Gobierno de los EE.UU. necesitaba ganar la paz en Afganistán. Para tal objetivo, los EE.UU. deben ganar los “corazones y las mentes" del pueblo afgano mediante la entrega de dividendos de paz a través de los auxilios y proyectos de desarrollo. ${ }^{39}$ Este es el principal motivo para la creación de los PRT. Éstos se inventaron para cerrar la brecha entre las operaciones en pro de la estabilidad dirigidas por militares y las actividades de reconstrucción dirigidas por civiles. ${ }^{40}$ Se espera que los PRT resuelvan el dilema de seguridad-desarrollo mediante la combinación de actividades en pro de la estabilidad por parte de los militares con las actividades de reconstrucción de los organismos de ayuda civil.

Los PRT son medidas transitorias destinadas a crear acceso humanitario a las personas que viven en zonas de alto riesgo civil, donde los organismos de ayuda no pueden o no quieren entrar debido a problemas de seguridad. Los PRT buscan cerrar la brecha seguridad-desarrollo mediante la entrega de dividendos de paz en un entorno no permisivo. Como agente para cerrar brechas, los PRT cumplen tres roles: (1) ser organismos que financian grupos locales, (2) ser directores de programas locales para implementar proyectos de desarrollo

39 A la comunidad militar le gusta utilizar la expresión “ganar los corazones y las mentes", pero este término se asocia estrechamente con operaciones psicológicas de los militares, que con frecuencia incitan reacciones alérgicas de la comunidad humanitaria. Los PRT deben evitar el uso de esta terminología y usar en su lugar otros términos, tales como ganar la aceptación local y lograr la seguridad humana para las poblaciones locales.

40 McNerney, Michael J., "Estabilización y reconstrucción en Afganistán: son los PRT un modelo o un desorden?”, en: Parámetros, invierno de 2005-06, p. 34. 
a nivel nacional y (3) ser agentes de implementación de proyectos de rápido impacto (QIP). El "componente civil" de los PRT, en calidad de directores de programas locales, puede asumir o facilitar grandes proyectos de desarrollo a largo plazo, que en pocos años evidencian dividendos de paz a la población. El primer y segundo rol de los PRT es cerrar tal brecha, mediante la financiación de contratos que entreguen "dividendos de paz "a través de QIPs y proyectos de desarrollo, y/o la ejecución de tales proyectos por sí mismos.

Por otro lado, el tercer rol ha recibido duras críticas por parte de la comunidad humanitaria, dado que si los PRT ejecutan proyectos QIP podrían socavar la labor de las agencias civiles y desdibujar el límite entre éstas últimas. Se puede argumentar que la entrega de dividendos de paz a las personas en zonas remotas a través de los QIP ejecutados por los PRT han demostrado ser un medio útil para cerrar la brecha seguridad-desarrollo cuando no hay una alternativa de capacidad civil disponible en la zona. ${ }^{41}$ De hecho, la Evaluación subrayó este punto afirmando que los PRT ofrecieron ayudas de reconstrucción humanitaria en zonas alejadas y violentas donde otros agentes de desarrollo no han sido capaces o no han estado dispuestos a operar..$^{42} \mathrm{El}$ Manual también señaló que los PRT tienen la capacidad de implementar proyectos de forma rápida y directa en comunidades remotas. ${ }^{43}$

Dicho esto, los QIPs no pueden ser un sustituto de los proyectos de desarrollo sostenible a largo plazo. El dilema seguridad-desarrollo no se puede resolver por sí solo a través de QIPs. Los PRT no son más que medidas de emergencia que pueden ayudar a mantener el impulso en la construcción de paz entre la población local manteniendo su confianza en el proceso por un tiempo. Así mismo, los PRT no deben ser un sustituto de las fuerzas de seguridad locales. Por lo tanto, es sumamente importante que la brecha seguridad-desarrollo se cierre del lado de la seguridad también. En este contexto, el pujante esfuerzo de los PRT por facilitar la SSR, especialmente en el entrenamiento de la ANP, es un paso significativo hacia la preparación para el traspaso de las tareas de seguridad a la autoridad local. Muchos PRT tienen instructores de la policía y/o tutores de la policía entre sus componentes civiles, y proporcionan formación a la ANP. Algunos PRT incluso han realizado patrullas conjuntas con la ANP.

41 La pregunta difícil aquí radica en cómo deben tratarse los QIPs ejecutados por los PRT que se llevan a cabo en parte para los propósitos de protección, y cómo debemos juzgar la conveniencia de tales QIP en una zona gris, es decir, en la que algunos organismos civiles están operando, a pesar de que la situación de seguridad sobre el terreno no es permisiva y la mayoría de los organismos civiles han decidido retirarse de la zona debido a problemas de seguridad.

42 Evaluación, p. 11.

43 Manual, p. 15. 
Los PRT también han ayudado en el programa de desarme, desmovilización y reinserción (DDR) y en el programa de "Cuarteles Temporales para Armamento Pesado", ${ }^{44}$ y mantiene su compromiso de ayudar a los programas de disolución de grupos armados ilegales (DIAG). La Evaluación también se reconoció que los PRT hicieron importantes contribuciones a la seguridad a través de su presencia, y mediante el apoyo a la ANP y el ANA, y el programa DIAG. ${ }^{45}$ En resumen, los PRT han contribuido positivamente a la creación de un entorno más estable en el que los organismos civiles, incluyendo las ONG, pueden trabajar sin restricciones graves de seguridad, especialmente en el Norte y el Este del país.

Sin embargo, no se debe confundir la principal labor de los PRT con la tarea de proveer seguridad. Los PRT no son proveedores de seguridad y por sí solos no puede garantizar la seguridad en la zona. De hecho, los PRT son más apropiados en un contexto de rango medio de violencia donde la inestabilidad todavía se opone a la participación amplia de las $\mathrm{ONG}$, pero donde la violencia no es tan aguda como para que predominen las operaciones de combate. ${ }^{46} \mathrm{El}$ proceso de construcción de paz en Afganistán todavía carece de una eficaz garantía de seguridad y la capacidad local para proporcionar seguridad humana para todos. Con el fin de cerrar la brecha entre la seguridad y el desarrollo, el pueblo afgano debe estar convencido de que los talibanes no regresarán después de que la ayuda internacional haya sido retirada, y debe estar seguro de que su vida será mejor en el marco del actual gobierno de que como lo fue en la época del régimen de los talibanes. Los PRT pueden ser una eficaz medida de transición para cumplir con algunas partes de estos requisitos, pero no son la panacea. Con el fin de ganar la paz en el Afganistán, necesitamos más PRTs y algo más que sólo PRTs.

\section{Conclusión}

Este documento ha asumido por objeto evaluar el desempeño de los PRT abordando la siguiente pregunta de investigación: ¿Han sido los PRT eficaces para cerrar la brecha civil-militar y la brecha seguridad-desarrollo en el proceso de construcción de paz en Afganistán?

44 Uesugi, Yuji, "Los equipos de reconstrucción provincial (PRT) y su contribución al DDR en Afganistán”, HIPEC Serie de Informes de Investigación $\mathrm{N}^{\circ} 3$ (marzo de 2006).

45 Evaluación, p. 11.

46 Evaluación, p. 6. 


\section{Brecha civil-militar}

La brecha entre civiles y militares fue recapitulada en el debate sobre el "espacio humanitario" o la eficacia de adherirse a los principios humanitarios en el establecimiento y el mantenimiento del acceso a las personas necesitadas de asistencia humanitaria. En este trabajo se ha afirmado que algunas diferencias en los principios de funcionamiento de los organismos humanitarios y las organizaciones militares provocó una gran tensión entre civiles y militares en Afganistán. Aunque las directrices actuales de relaciones civiles y militares hacen hincapié en separar la asistencia humanitaria de las operaciones militares, los Principios Rectores de los PRT, en esencia, promueven la integración civilmilitar para generar unidad de esfuerzos entre los diversos agentes del terreno. Si bien la mayoría de los elementos de los Principios Rectores de los PRT indican la permanencia dentro de los parámetros de las actuales directrices para las relaciones entre civiles y militares, el enfoque fundamental de "integración" civil-militar en el concepto de los PRT contradice el argumento central de la "separación" en las directrices existentes. Este documento ha explorado por qué los organismos humanitarios han sido precavidos acerca de desdibujar el límite entre ellos y las organizaciones militares.

Este artículo subraya la importancia de preservar el "espacio humanitario", y argumenta que los PRT podrían ser un instrumento eficaz para el cierre de la brecha civil (humanitaria)-militar en una situación en la que los organismos humanitarios no puedan funcionar debido a la pérdida del espacio humanitario. Además, los PRT pueden ser una herramienta útil, incluso en una situación en la que los organismos humanitarios estén funcionando de manera eficaz, realizando funciones complementarias tales como la facilitación de SSR y DDR, y apoyo a grandes proyectos de infraestructura. Este documento ha llegado a la conclusión, sin embargo, de que la falta de capacidad y recursos civiles dentro de los PRT, junto con la mala relación entre estos últimos y la comunidad humanitaria, han impedido el ejercicio de su pleno potencial como mecanismo para cerrar brechas.

\section{Brecha seguridad-desarrollo}

Este artículo sostiene que el concepto de PRT puede caracterizarse como la integración civil-militar y se inventó para hacer frente a la brecha seguridaddesarrollo que surgió en Afganistán luego de la caída del régimen talibán. Se puede concluir que los PRT comenzaron a cerrar dicha brecha mediante la participación en actividades de emergencia tales como la promoción de SSR y de DDR, la entrega "dividendos de paz" a través de QIP y de proyectos de desarrollo. Como un agente para cerrar brechas, los PRT tiene tres roles: (1) ser 
organismos que financian grupos locales, (2) ser directores de programas locales para implementar proyectos de desarrollo a nivel nacional, y (3) ser agentes de implementación de QIPs. Los PRT han demostrado ser un medio útil para cerrar la brecha seguridad-desarrollo cuando no hay una alternativa de capacidad civil disponible en la zona.

Después de todo, los QIPs no son más que medidas de emergencia que pueden ayudar a mantener el impulso en la construcción de paz entre la población local y puede mantener su confianza en el proceso por un tiempo. Sin embargo, los QIPs no deben ser un sustituto de los proyectos de desarrollo sostenible a largo plazo. El dilema seguridad-desarrollo no se puede resolver por sí a través de QIPs solamente. Asimismo, los PRT no deben ser un sustituto de las fuerzas de seguridad. Por lo tanto, el pujante esfuerzo de los PRT por facilitar el restablecimiento de la seguridad (SSR), especialmente en el entrenamiento de la ANP, es un paso significativo hacia la preparación para el traspaso de las tareas de seguridad a la autoridad local.

\section{El gobierno es clave}

A diferencia de los organismos humanitarios cuyos objetivos principales se centran en evitar la crisis humanitaria y aliviar el sufrimiento humano, el objetivo de la construcción de la paz es mucho más amplio y más global. La naturaleza compleja de la construcción de paz, en la que los PRT fueron diseñados para operar, hace un llamado a un enfoque más comprensivo e integrado para generar efectos deseados y producir unidad de esfuerzos entre las diversas iniciativas.

Aunque los PRT han por lo menos tratado, y en algunos casos logrado, cerrar las brechas civil-militar y seguridad-desarrollo en el proceso de paz en Afganistán, los PRT no han abordado el urgente problema del gobierno en Afganistán. El concepto original de los PRT estipula que su modelo solicita la integración de los tres aspectos fundamentales de la consolidación de la paz: la seguridad, el desarrollo y el gobierno. Al mismo tiempo, los PRT son operaciones de estabilización incapaces de de asumir tareas más amplias de construcción de paz, tales como el buen gobierno, el Estado de derecho, la administración pública y así sucesivamente. Es cierto que algunos PRT ayudaron extender la autoridad del gobierno central, proporcionando apoyo técnico y organización a los gobernadores de las provincias y a los ministerios, como se indica en la evaluación realizada, ${ }^{47}$ pero éstos no tienen un medio eficaz para desarrollar la capacidad local de gobernar.

Evidentemente, una de las piezas más importantes que hace falta en el proceso de construcción de paz en Afganistán, identificada en varias ocasiones, 
es la presencia efectiva de los gobiernos locales, tanto a nivel nacional como provincial. La falta de capacidad local para llevar a cabo las tareas de gobierno y convertirse en actores responsables ha socavado la labor de los PRT. Este no es un problema nuevo, único en el proceso de paz de Afganistán. De hecho, la comunidad internacional ha experimentado problemas similares en otros procesos de construcción de paz, como en Timor Oriental, Sierra Leona, Liberia y otros países. Sin embargo, el problema es especialmente grave y más difícil en Afganistán, ya que los expertos en "gobierno" no han podido entrar en provincias remotas debido a la situación de seguridad en ese terreno.

La comunidad internacional está empezando a abordar este problema mediante la vinculación en los PRT de civiles expertos en el ámbito del buen gobierno, el Estado de derecho, y la administración pública, pero la comunidad internacional no ha podido hallar una fórmula mágica para encontrar civiles expertos en casa. El Equipo País de las Naciones Unidas en Afganistán, y en particular el UNDP, ${ }^{48}$ deberían tener la capacidad civil para hacer frente a la difícil tarea de desarrollar el buen gobierno local. Los PRT necesitan mejorar su relación con la UNAMA y el Equipo País de Naciones Unidas en este aspecto. $\mathrm{El}$ reto es que los PRT adopten un enfoque en el que las actividades civiles de cada PRT estén dirigidas por una nación líder sobre bases, pero no ha habido ningún mecanismo dentro de los PRT que permita al personal de las Naciones Unidas asumir las funciones civiles críticas, aunque la ISAF de Naciones Unidas sea una operación de paz bajo mandato establecido por la Resolución del Consejo de Seguridad de la ONU.

En los últimos cinco años de amarga experiencia en Afganistán se demostró que se requiere más que la entrega de dividendos de paz mediante el desarrollo de proyectos para realmente lograr la paz en Afganistán. Los PRT no pueden ganar los corazones y las mentes de la población local o su aceptación simplemente ejecutando QIPs y otro tipo de proyectos de desarrollo. Estos últimos por sí solos no pueden generar confianza en la población con respecto a la actual administración. La actual administración, tanto a nivel nacional como provincial, tiene que demostrar que es eficaz y que, por lo tanto, el actual proceso de paz traerá estabilidad y un mejor futuro. Para lograr la paz en Afganistán, el actual proceso de construcción de paz debe ser visto por el pueblo afgano como confiable y el actual gobierno debe ser considerado como legítimo. De hecho, el desarrollo no tiene sentido sin el gobierno, y por supuesto el gobierno sin desarrollo es inalcanzable. De la misma manera podemos decir que la gobernabilidad sin seguridad es inalcanzable y sin sentido. Hasta ahora, la comunidad internacional ha creado los PRT para cerrar las brechas civiles-militares y seguridad-desarrollo, 
pero no ha sido capaz de abordar la brecha seguridad-gobierno, o la brecha de la capacidad local. De hecho, el desarrollo de la capacidad local es un elemento clave para la exitosa entrega de la responsabilidad de construcción de paz a las autoridades locales y, por tanto, una efectiva estrategia de transición para los PRT, pero nadie está ahí en las remotas provincias, a excepción de los mal equipados PRT. 